\title{
Les maladies des mollusques bivalves : risques et prévention
}

\author{
H. Grizel \\ Institut français de recherche pour l'exploitation de la mer (IFREMER), Station de Sète, 1 rue Jean Vilar, \\ B.P. 171. 34203 Sète Cedex, France
}

\begin{abstract}
Résumé
La conchyliculture basée sur les activités de pêche et d'élevage dans le milieu naturel s'est considérablement développée au cours de ces dernières années, notamment grâce à la production de naissain dans les écloseries et les nurseries. L'accroissement des demandes des échanges de produits, facilité par les moyens modernes de transport, a provoqué une augmentation des risques de propagation de maladies. En s'appuyant sur des exemples, une analyse est faite sur les risques encourus liés aux pratiques conchylicoles et à l'état actuel des connaissances scientifiques. Des recommandations sont formulées sur la prévention, sur le besoin de réglementations et sur les difficultés rencontrées dans ce domaine. Enfin, quelques suggestions sont proposées pour mieux gérer l'antagonisme existant entre la demande des marchés et la nécessité de préserver la conchyliculture.
\end{abstract}

\section{Mots-clés}

Analyse des risques - Commerce international - Conchyliculture - Maladies Mollusques bivalves - Santé animale.

\section{Introduction}

La production de mollusques bivalves est une activité traditionnelle, très ancienne, répartie dans plusieurs régions du monde, en particulier dans l'hémisphère nord. Depuis plusieurs années, cette activité qui concerne la pêche et, de plus en plus, lélevage, met sur le marché environ 5,5 millions de tonnes de produits. Les huîtres, notamment l'huître creuse (Crassostrea gigas), les moules (Mytilus edulis, M. galloprovincialis et Perna viridis), les coquilles Saint-Jacques (Pecten jacobeus, P. maximus) et les pétoncles (Patinopecten yessoensis, Argopecten iriradians), les clams (Mercenaria mercenaria, Ruditapes philippinarum et Tapes decussatus) et les coques (Anadara granosa, Cardium edule) sont les principales espèces produites et consommées (12) Animaux filtreurs, généralement sessiles, les mollusques bivalves nécessitent, chaque jour, d'être immergés pendant de longues périodes afin de croître et de fabriquer des substances de réserve dont ils ont besoin pour traverser des périodes sensibles, comme celle de la reproduction. Ces caractéristiques physiologiques, dont notamment le fait d'être filtreur, leur conferent des avantages et des inconvénients en tant qu'animaux intéressant l'aquaculture. En effet, hormis lors des premières phases d'élevage qui peuvent ètre conduites en écloserie et en nurserie, les cycles d'élevage des mollusques bivalves s'effectuent dans le milieu naturel où ils prélèvent directement leur nouniture. Ce poste qui occupe généralement une place importante dans le calcul des coûts de revient des autres types d'activités aquacoles (pisciculture, pénéiculture) est, dans ce cas, quasiment nul. En contrepartie, les mollusques bivalves sont plus sensibles aux conditions environnementales, notamment aux conditions sanitaires et zoosanitaires qui auront des influences directes sur leur qualité sanitaire et sur leur état de santé.

Seules les maladies spécifiques des moliusques, qui ont des répercussions significatives et durables sur les élevages seront considérées ici. Afin de bien identifier les risques et d'examiner les solutions envisageables pour la prévention, nous décrirons, d'abord, les méthodes d'élevages et les procédés de préparation pour la mise en vente de quelques espèces de coquillages; nous ferons ensuite une synthèse des connaissances acquises sur les maladies et les agents pathogènes majeurs. 


\section{Les principales méthodes de capture et d'élevage des mollusques bivalves}

\section{La pêche}

La péche des coquillages peut être sauvage, c'est-à-dire pratiquée n'importe où et sans être soumise à une réglementation et à des contrôles. Ce mode de pêche généralement prohibé existe, de fait, dans la grande majorité des pays. Les produits issus de cette pêche sont destinés à la consommation personnelle ou bien sont réintroduits dans les circuits d'élevage et de commercialisation dans le même secteur ou dans d'autres secteurs géographiques.

Dans le cas des autres formes de pêche, dites réglementées, les coquillages sont prélevés sur des gisements naturels répertoriés et classés. L'ouverture de la pêche s'effectue pendant des durées limitées et contrôlées.

L'état sanitaire el zoosanitaire de ces coquillages est aussi généralement connu, bien que, dans de nombreux pays, l'expérience révèle que très peu de données scientifiques sont disponibles dans ce domaine. Comme dans le premier cas, les produits issus de la pêche peuvent être réimmergés dans différents endroits, y compris dans d'autres pays, notamment lorsque la pêche conceme des jeunes individus destinés à être reparqués.

Les techniques de pêche dépendent des pays, de la surface des gisements et des espèces. Les principaux modes de pêche sont la pêche à pied ou depuis une embarcation. Dans ce dernier cas, les techniques employées peuvent être classées en trois catégories: manuelles (utilisant par exemple la pince à coquillages), tractées (par exemple au moyen de dragues) et par aspiration (par exemple en utilisant une suceuse). L'impact direct de ces techniques sur la santé des cheptels n'est pas clairement établi ; il est toutefois connu qu'elles ont des répercussions plus ou moins importantes sur le biotope, ainsi que sur les coquillages eux-mêmes (enfouissement, bris de coquilles, stress). Dans ce dernier cas, des effets indirects peuvent être induits, par une fragilisation des coquillages.

\section{La production de naissain ou juvéniles}

La maitrise compiète du cycle d'élevage des coquillages est basée sur l'approvisionnement en juvéniles ou naissain. Ces derniers peuvent être collectés dans le milieu naturel ou bien produits dans des écloseries et des nurseries (25).

Dans le premier cas, la collecte s'effectue par pêche directe de juvéniles (voir ci-dessus) ou bien par la pose dans le milieu naturel de collecteurs appropriés pour le captage et la fixation des larves pédivéligères. Les principaux collecteurs employés pour les huitres creuses ( $C$. gigas) sont des coquilles d'huîtres creuses ou de coquilles Saint-Jacques, des tubes, des "chapeaux chinois " ou des cadres ajourés en matiere plastique. D'autres techniques plus ou moins élaborées existent pour d'autres espèces de coquillages. Dans tous les cas, les collecteurs sont mis à l'eau au cours de la période de reproduction et ils y séjournent de trois à neuf mois. Le naissain issu de cette récolte peut être commercialisé et réimmergé pour l'élevage, soit après séparation du collecteur (opération de détroquage), soit sur le collecteur.

Dans le deuxième cas, la production de naissain a lieu en écloserie, c'est-à-dire dans un milieu contrôlé. Le principe de l'écloserie repose sur la maîtrise de la reproduction des coquillages et de l'élevage des larves qui comprennent plusieurs opérations importantes

- la création et la gestion des stocks de géniteurs,

- la maturation contrôlée des géniteurs,

- le déclenchement de la ponte et la surveillance des conditions de fécondation,

- la réalisation des élevages larvaires (nourriture appropriée, densité, température, conditions sanitaires, etc.),

- la production de nourriture adéquate pour obtenir une bonne maturation des géniteurs et un bon développement larvaire,

- la métamorphose et la fixation, lorsque cela est nécessaire pour les larves,

- l'élevage des microjuvéniles.

Ces derniers sont, par la suite, élevés dans des nurseries en système intensif, avec addition ou non de noumiture (phytoplancton). Les nurseries nécessitent la disponibilité de grands volumes d'eau, prélevés daans des zones riches en nourriture adéquate pour les juvéniles. Lorsque les juvéniles atteignent une taille jugée suffisamment intéressante et rentable pour les éleveurs, ils sont commercialisés pour l'élevage en milieu ouvert.

Le télécaptage, mis au point depuis une quinzaine d'années et applicable surtout aux huîtres, est un procédé intermédiaire entre les deux précédents. En effet, il bénéficie des possibilités de production en quantité importante de larves ceillées (stade de prémétarnorphose) qui peuvent être expédiées dans de bonnes conditions, rapidement et à moindre coût vers des zones éloignées du lieu initial de production. Remises à l'eau dans des enceintes fermées et soumises à des conditions adéquates (nourriture, température, densité), ces larves peuvent alors se fixer sur les collecteurs qui sont immergés dans les enceintes. Après la fixation des larves et suite à une phase d'acclimatation progressive, notamment à l'égard de la temperature, les collecteurs garnis de naissain sont placés dans des zones de prégrossissement situées en milieu ouvert. Ce procédé permet de réduire les coûts de production des juvéniles, en particulier ceux inhérents au passage en nurserie. et de satisfaire la demande qualitative et quantitative de l'éleveur. 


\section{Les procédés et les techniques d'élevage}

Malgré la diversité des pays producteurs et du nombre d'espèces concemées, les procédés d'élevage restent peu nombreux et peu diversifiés. Pour les coquillages fouisseurs (palourdes, coques, clams), les paramètres les plus importants à définir se rapportent à la nature du sol (granulométrie), à l'exposition et au degré bathymétrique du parc et, en fonction de la taille des juvéniles, à la densité et à la protection des semis. Pour les non-fouisseurs, trois grands procédés, présentant des variantes techniques, sont recensés: les élevages à plat sur sol, les élevages en surélévation et les élevages en suspension. Les premiers sont applicables aux huîtres et aux moules et peuvent être conduits, selon les espèces, sur des parcs découvrants lors des basses mers ou bien sur des parcs situés en eau profonde $(-4 \mathrm{~m}$ à $-10 \mathrm{~m}$ de profondeur). Dans ce dernier cas, pour être rentable, l'exploitation doit attribuer de grandes surfaces de parcs et utiliser des bateaux bien équipés

Les élevages en surélévation s'appliquent surtout aux espèces dont la répartition naturelle se situe sur l'estran de basse mer, comme les huitres de palétuviers et plusieurs espèces du genre Crassostrea. Les poches ou casiers en plastique, les casiers de bois posés sur des tables en fer ou en bois pour les huîtres et pour les moules, les pieux de bambou ou de chêne plantés dans le sol, restent les techniques les plus courantes.

Les élevages en suspension ont éré développés en pleine mer, dans des endroits plutôt abrités. Des cordes sur lesquelles sont maintenus les coquillages (huitres, moules, coquilles Saint-Jacques et pétoncles) sont suspendues à des superstructures («bateas", radeaux, tables fixes) ou à des longues lignes.

Quel que soit le procédé utilisé, l'élevage des mollusques devrait reposer sur plusieurs grands principes, parmi lesquels peuvent être soulignées

- la disponibilité de naissain ou de juvéniles d'une taille suffisante pour la mise en élevage et qui soient indemnes de maladies,

- l'identification de sites abrités avec des biotopes correspondant aux besoins des espèces à élever (par exemple la nature des sols, le gradient de leur température et de salubrité, la productivité primaire),

- la connaissance de la qualité sanitaire et de l'état des pollutions des eaux,

- la présence d'infrastructure à proximité des sites d'élevage.

\section{La commercialisation des coquillages}

La commercialisation des coquillages conceme tous les stades d'évolution, depuis les larves jusqu'aux adultes de taille commerciale. De nombreux courants commerciaux existent à l'intérieur d'un pays, mais également entre pays de continents différents.

Le développement des écloseries et l'efficacité des transports aériens ont provoqué un accroissement très sensible des échanges de mollusques entre les pays. Des écloseurs américains ont ainsi déclaré avoir exporté plus de 400 millions de naissain de $C$. gigas par an (Tableau I) (7). Pour l'Europe, Le Borgne (22) fait état d'un mouvement annuel de 10 à 20 millions de naissain de palourdes européennes (Ruditapes decussatus) depuis la Grande-Bretagne et la France vers l'Espagne et le Portugal et de 100000 millions de larves et naissain d'huitre creuse depuis la Grande-Bretagne et la France vers l'Irlande.

\section{Tableau 1}

Exemples d'échanges internationaux de juvéniles de mollusques depuis la façade ouest de l'Amérique du Nord (7)

\begin{tabular}{lll}
\hline Espèces & Stade & Continents destïnataires \\
\hline $\begin{array}{l}\text { Gastéropode } \\
\text { Haliotis spp. (ormeau) } \\
\text { Bivalves }\end{array}$ & naissain & Amérique du Sud et Asie \\
Crassostrea gigas (huittres) & larves & $\begin{array}{l}\text { Amérique du Sud, Asie, Afrique } \\
\text { et Europe }\end{array}$ \\
$\begin{array}{l}\text { Tapes spp. (clams) } \\
\text { Mytilus spp. (moules) }\end{array}$ & naissain & Amérique du Sud, Asie et Europe \\
\end{tabular}

Les transferts de mollusques de taille commerciale, à l'état vivant, sont plus restreints, car le coût du transport devenu beaucoup plus élevé grève le coût de revient et par conséquent le prix de mise en vente de ces produits. Seules des espèces considérées comme un produit de luxe, ou très prisées, sont réellement sujettes à des échanges internationaux. Par contre, de nombreuses relations commerciales ont été établies entre les pays ayant développé des échanges par transport routier. C'est le cas de plusieurs pays européens qui, en fonction de la disponibilité locale de coquillages, en importent depuis des pays voisins (Tableau II).

\section{Tableau II}

Exemples d'échanges de mollusques de taille commerciale entre pays européens

\begin{tabular}{lll}
\hline Espèces & Pays d'origine & Pays destinataires \\
\hline $\begin{array}{l}\text { Ostrea edulis } \\
\text { (huître plate) }\end{array}$ & $\begin{array}{l}\text { Croatie } \\
\text { Grèce }\end{array}$ & $\begin{array}{l}\text { Italie, puis France } \\
\text { Italie, puis Espagne }\end{array}$ \\
Moules & $\begin{array}{l}\text { Espagne } \\
\text { Pays-Bas } \\
\text { Irlande }\end{array}$ & France \\
& $\begin{array}{l}\text { Irlande } \\
\text { Crassostrea gigas } \\
\text { (huitre creuse) }\end{array}$ & France \\
\hline
\end{tabular}


Ces coquillages vivants sont quelquefois destinés à la consommation directe, mais dans la plupart des cas, ils sont réimmergés en bassin ou reparqués avant d'être commercialisés. La réimmersion est également pratiquée afin de permettre à des mollusques importés d'atteindre la taille commerciale.

Les mollusques sont également commercialisés à l'état décoquillé, congelé, surgelé, en boîte ou conditionnés en plats cuisinés. Ce mode très répandu aux États-Unis d'Amérique, dans plusieurs pays européens ainsi qu'en Asie du Nord et du Sud-Est ne pose évidemment pas de problèmes particuliers pour la contamination des coquillages; par contre, toutes ces transformations requièrent des précautions spécifiques afin de garantir la mise sur le marché de produits salubres.

\section{La notion de principales maladies infectieuses des mollusques bivalves}

Les notions de principales maladies infectieuses et conséquemment de principaux agents pathogènes affectant les mollusques bivalves restent controversées dans le monde scientifique, chaque auteur ayant d'une manière générale tendance à vouloir faire considérer comme important l'agent quiil a décrit et à préserver l'activité conchylicole de son pays de toute introduction d'agents pathogènes $(7,18)$.

Cependant, comme le souligne Elston (7), le risque zéro n'existe pas dans ce domaine. L'alternative reste soit l'adoption d'un "pseudo-risque zéro $»$, avec l'interdiction officielle de toutes transactions commerciales de mollusques vivants, soit celle d'un risque " $x$ » difficilement évaluable, comme nous le verrons ultérieurement.

A travers un cas historique, particulièrement riche d'enseignements, nous tenterons de souligner les difficultés inhérentes à l'établissement de règles satisfaisantes, reconnues et surtout applicables.

\section{Cas de Bonamia ostreae}

Le premier document relatif à Bonamia, nommé alors microcells, a été publié aux États-Unis d'Amérique (20), les auteurs associant simplement une faible mortalité d'huîtres plates à la présence de microcellules dans les hémocytes.

En 1969, les informations disponibles étaient donc extrêmement succinctes et pour des raisons probablement d'ordre économique, les microcells n'ont pas fait l'objet d'écudes complémentaires portant sur la morphologie, l'ultrastructure, l'infectiosité et l'épidémiologie en général. Ce n'est qu'en 1986 (8) qu'une autre publication américaine fait état de quelques données supplémentaires concernant Bonamia sp., faisant suite à de nombreux travaux réalisés en Europe $(13,14,28,29,34,35,36,37,38)$. Entre ces deux dates, quelques ostréiculteurs français, pensant bien faire pour diversifier leur approvisionnement en naissain, ont introduit, en France, des juvéniles dhuitres plates produits dans des écloseries califomieńnes. Ces lots avaient été certifiés indemnes de maladies auprès des ostréiculteurs concernés. Le résultat de ces immersions frauduleuses, accomplies uniquement, d'une part, sous la pression commerciale des écloseurs américains, d'autre part, en raison de l'inconscience d'ostréiculteurs français, a été catastrophique pour les élevages d'huitres plates européennes. En elfet, dès 1979, des examens histologiques d'huîtres subissant des taux de mortalité importants permettaient de metre en évidence un agent pathogène intrahémocytaire et d'en décrire l'ultrastructure (29). Par la suite, les nombreux travaux effectués rapidement sur Bonamia ostreae ont permis à la communauté scientifique d'en connaître l'évolution géographique $(13,34,35,36,38)$ et d'en préciser les modalités d'infection (37). La reproduction de la maladie au laboratoire, les tests de sensibilité d'autres espèces dhuitre plate $(1,14,28)$, le rôle de paramètres d'élevage dans le développement de la maladie (11) ont été d'autres progrès réalisés.

Par ailleurs, depuis les années 1970, les nombreux examens histologiques effectués en permanence chez la plupart des espèces d'élevage sur presque tout le littoral français, ont permis de conclure à la spécificité de B. ostreae pour l'huître plate, les autres coquillages (huitres, moules) n'étant pas des hotes porteurs sains (31). Malgré le: fait que ces informations sur les risques encourus à importer des huitres plates françaises étaient rendues publiques, les échanges traditionnels perdurèrent quelques années entre la France, !'Espagne et les Pays-Bas, permettant ainsi la propagation de la maladie. Celle-ci, probablement à cause d'introductions opportunistes, a également gagné la Grande-Bretagne (2) et l'Irlande (24). Ultérieurement, elle a été encore signalée ponctuellement dans d'autres pays (Danemark, Italie)

Cet historique soulève des questions importantes et pose plusieurs problèmes fondamentaux.

La première question est relative à l'analyse de l'information disponible et à sa véracité : comment peut-on juger de l'importance ou non d'une maladie ou d'un agent pathogene lorsque la litterature nioffre quasiment pas d'informations?

Dans le cas présent, les importations étaient interdites (absence d'agrément entre la France et les États-Lnis d'Amerique), mais comment aurait-on considéré cette maladie en cas d'agrément ? Compte tenu des paramètres utilisés pour établir la liste des maladies à declaration obligatoire, les microcells n'auraient pas été retenus comme agent d'une maladie de cette catégorie, car, d'apres la littérature existant en 1969, ils provoquaient de faibles taux de mortalité, dans un espace géographique limité (7). 
D'un autre côté, si l'on avait retenu, à l'origine, ces microcells comme agents pathogènes déclarables, il n'y aurait eu alors aucune raison pour ne pas retenir tous les agents décrits dans la littérature, plus ou moins associés à un épisode de mortalité.

Cette réflexion illustre les difficultés inhérentes aux décisions préventives. Celles-ci en effet sont soit laxistes, et peuvent alors devenir catastrophiques, soit trop prudentes et sont alors extrêmement contraignantes et risquent d'être jugées inopportunes par les conchyliculteurs. D'autres publications ont décrit l'association d'agents pathogènes, comme des rickettsies (5), à des épisodes de mortalité ou des haplosporidies (cas de C. gigas) (25) qui n'ont eu, malgré le transfert de coquillages, aucune conséquence ultérieure et n'ont pas engendré d'épizooties, ni chez l'espèce concemée, ni chez d'autres espèces.

La deuxième question découle de la précédente : quel statut doit-on donner à un agent pathogène associé à l'apparition d'une mortalité anormale et pour lequel très peu d'informations sont disponibles?

Compte tenu du cas de Bonamia, il parait important que le pays souhaitant exporter sache répondre aux demandes du pays importateur, afin que celui-ci soit en mesure de juger, en connaissance de cause, du risque qu'il estime pouvoir prendre. La présence d'un agent pathogène ne devant pas systématiquement devenir un moyen aisé d'établir des barrières aux échanges commerciaux, il paraît nécessaire de définir, au préalable, des protocoles scientifiques réalistes, concernant l'acquisition de données minimales sur l'agent et sur l'épidémiologie. Ceci suppose, lorsqu'un agent infectieux est observé, au minimum trois ans de travail sérieux sur le cas, pour pouvoir établir un pronostic sur son infectiosité ou sa virulence, sur sa transmission et sur son importance. Pendant cette période, les échanges concernant l'espèce porteuse devraient être interdits entre les pays. Le corollaire devrait être que les pays qui ne font pas l'effort pour acquérir ces données minimales se verraient contraints à limiter leurs exportations aux seuls coquillages indemnes de toute affection.

Un autre point important, complémentaire des précédents, concerne la publication des résultats et les garanties que peut donner un pays et ses autorités compétentes sur la situation zoosanitaire des mollusques qu'il produit. En effet, il est reconnu que plusieurs pays, pour des raisons politico-économiques, font de l'obstruction à la publication de résultats scientifiques concernant les maladies ou les déclarations de mortalité sur leur territoire. D'autres pays, pour des raisons économiques, n'ont ni la capacité ni la compétence nécessaires pour assurer un suivi zoosanitaire de base et pour intervenir efficacement lorsque des cas anormaux de mortalité surviennent. Ces situations se traduisent par un affichage erroné de la réalité, ces pays déclarant être indemnes de maladies. À contrario, les professionnels conchylicoles des pays où les scientifiques mettent leurs résultats à la disposition de la communauté intemationale subissent des baisses de vente, car ils sont contraints de réduire leurs exportations.
Ces faits sont également largement reliés à l'effort d'échantillonnage et de contrôle que consent chaque pays. Ainsi, en Europe, l'accroissement du nombre d'analyses et l'amélioration des plans d'échantillonnages, pour la mise en conformité avec la Directive européenne 91/67, ont permis de mettre en évidence la présence de $B$. ostreae dans plusieurs zones géographiques de Grande-Bretagne et d'Irlande, réputées jusqu'alors indemnes de bonamiose.

Cet exemple, non seulement illustre les difficultés décisionnelles autour des maladies naissantes (comme cela a été le cas pour l'encéphalopathie spongiforme bovine) mais souligne également les oppositions d'intérêts qui peuvent exister entre le besoin exprimé de favoriser les échanges commerciaux et celui tout aussi légitime de protéger ses propres productions. Il met également en lumière l'important besoin en matière de contrôle permanent et de recherche, afin de fournir aux professionnels et aux autorités compétentes les meilleures informations dans le minimum de temps. Enfin, il éclaire bien la notion relative de principales maladies infectieuses. Dans le cas des propositions faites par l'Office intemational des épizooties (OIE) pour établir un Code stipulant des règles à suivre, la liste des maladies à déclaration obligatoire a été établie en prenant compte des considérations issues des expériences et des données de la littérature, mais également des pratiques courantes de la profession (26).

Ainsi, pour le choix des maladies déclarables, il a été tenu compte de l'importance de l'impact de la maladie sur l'économie conchylicole $(11,16)$, de la qualification de la maladie (épizootie, zoonose, etc.), de sa clurée, de sa couverture géographique, de son infectiosité et cle son spectre de spécificité (16, 17, 18). Les experts ont également tenu compte de la présence ou non d'agents infectieux (et n'ont donc pas inscrit les maladies à étiologie non confirmée telles que la maladie de Malpèque), ainsi que de la disponibilité de méthodes de diagnostic, incluant l'histologie comme telle, pour effectuer raisonnablement les contrôles. Enfin, ils ont jugé opportun de spécifier sous le nom générique de la maladie, les agents pathogènes effectivement déclarables, car au sein d'un même genre, certaines espèces sont bénignes ou sans importance pour l'économie. La liste actuellement proposée en tenant compte de tous ces critères est rappelée dans le Tableau III.

\section{Tableau III}

Maladies des mollusques à déclaration obligatoire à l'Olfice international des épizooties (26)

\begin{tabular}{ll}
\hline Maladies & Agents pathogènes concernés \\
\hline Bonamiose & $\begin{array}{l}\text { Bonamia ostreae, Bonamia spp. (Australie, Nouvelle- } \\
\text { Zélande) }\end{array}$ \\
Haplosporidose & Haplosporidium nelsoni et $H$. costale \\
Marteiliose & Marteilia refringens et $M$. sydneyi \\
Mikrocytose & Mikrocytos mackini et $M$. roughleyi \\
Perkinsiose & Perkinsus marinus et $P$. olseni \\
\hline
\end{tabular}


Cette liste reste évidemment évolutive et pourra être complétée, dans le futur, avec des agents pathogènes confirmés répondant aux critères retenus pour le choix.

\section{La notion de porteurs sains}

La notion de porteurs sains est importante à prendre en compte car comme nous l'avons décrit précédemment, les élevages de mollusques s'effectuent dans un milieu ouvert au sein duquel plusieurs espèces, susceptibles d'être transférées, cohabitent. Cette notion va donc au-delà de la simple spécificité et rejoint la notion d'hôte réservoir passif. Ce point, rarement pris en considération de manière formelle, a pris de l'importance lors des discussions entre les experts des pays de l'Union européenne et ceux de l'OIE et du Conseil international pour l'exploration de la mer (CIEM).

Les débats sur ce sujet ont permis de mettre en lumière la faiblesse des données de la littérature, et la voie offerte aux autorités compétentes des Pays Membres de se prémunir abusivement de toute importation. Ils ont également souligné le besoin institutionnel de définir des protocoles scientifiques minimum et maximum, permettant de statuer sur le rôle de porteur sain ou non de l'espèce à transférer. En effet, l'absence de tels protocoles modèles officiels, a suscité des demandes excessives, en coût et en temps, de la part de pays susceptibles de recevoir ces hôtes jugés non porteurs. Les membres du Groupe de travail du CIEM sur les maladies des organismes marins, qui s'est préoccupé de ce problème, ont déposé une proposition concrète et réaliste (Fig. 1).

Celle-ci a le mérite de permettre la réalisation d'une étude concrète, logique et informative à moindre coût en offrant un maximum de garanties, pour l'analyse du risque.

Lapplication de ces protocoles pour déterminer le statut de C. gigas, de M. galloprovincialis et de M. edulis à l'égard de B. ostreae et de Marteilia refringens aurait pu permettre daboutir plus vite à certaines conclusions. Toutefois, il est important de noter qu'après bien des discussions, l'huitre creuse ( $C$. gigas) a été reconnue non porteuse pour ces deux agents pathogenes (31). Dans le cas de $M$. refringens, la prévalence extrêmement faible (de l'ordre de 0,001\%), l'observation dans les épithéliums digestifs uniquement de rares jeunes stades qui n'évoluaient pas et l'examen rétrospectif de certaines données historiques ont permis au groupe d'experts de considérer le risque encouru comme quasiment nul.

Pour l'avenir, il sera utile de se reporter à la proposition du CIEM qui a, entre autres mérites, celui de prendre en compte l'expérience d'équipes ayant travaillé de manière permanente sur le contrôle et l'étude des maladies de mollusques.

Dans la liste des hôtes des agents pathogènes à déclaration obligatoire auprès de l'OIE, le groupe d'experts a pris en compte ces considérations et a proposé d'inclure, en plus de

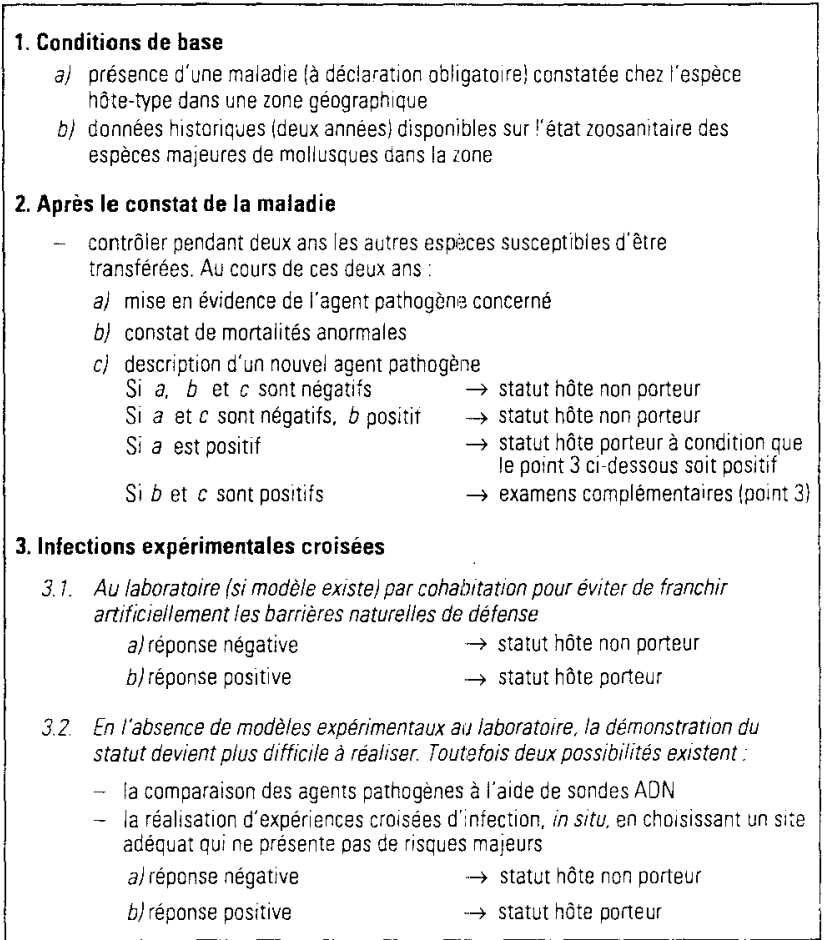

Fig. 1

Schéma expérimental et décisionnel pour établir le statut d'hôte porteur

lhôte spécifique, initialement rapporté comme tel dans la littérature, les hôtes réceptifs et sensibles à la maladie, contaminés naturellement ou dans des conditions expérimentales douces (cohabitation) $(1,17,18)$ (Tableau IV).

\section{Tableau IV}

Liste des hôtes des agents pathogènes des maladies à déclaration obligatoire (26)

\begin{tabular}{|c|c|c|}
\hline Agent pathogène & Hôte & Hôtes expérimentaux \\
\hline Bonamia ostreae & Ostrea edulis & $\begin{array}{l}\text { O. Lurida, } 0 \text { angasi, } \\
\text { O. puelchana, } \\
\text { O. denselamellosa et } \\
\text { Tiostrea chilensis }\end{array}$ \\
\hline Bonamiasp. & $\begin{array}{l}\text { Tiostrea chilensis } \\
\text { Ostrea angasi }\end{array}$ & \\
\hline \multicolumn{3}{|c|}{ Haplosporidium nelsoni Crassostrea viryinica } \\
\hline \multicolumn{3}{|l|}{ Haplosporidium costale } \\
\hline Marteilia refringens & Ostrea edulis & $\begin{array}{l}\text { O. angasi, } 0 \text {. puelchana, } \\
\text { O. denselamellosa et } \\
\text { T. chilensis }\end{array}$ \\
\hline Marteilia sydneyi & Saccostrea commercialis & \\
\hline Mikrocytos mackini & Crassostrea gigas & $\begin{array}{l}\text { C. virginica, } 0 \text { edulis, } \\
\text { O. conchaphila }\end{array}$ \\
\hline Mikrocytos roughleyi & Saccostrea commercialis & \\
\hline Perkinsus marinus & C. virginica & nombreux autres mollusques \\
\hline Perkinsus olseni & Haliotis spp. & nombreux autres mollusques \\
\hline
\end{tabular}


Cette liste est également évolutive, le statut de plusieurs hôtes affectés par des protozoaires du genre Marteilia devant être prochainement précisé.

\section{Analyse des risques}

\section{Les risques liés aux conditions d'élevage}

La conchyliculture pratiquée en milieu ouvert et non contrôlable est une activité très fragile exposée aux risques de maladies. Son mode de fonctionnement, qui repose la plupart du temps sur des échanges d'animaux à des stades différents de leur vie, constitue un des risques majeurs d'introduction et de propagation d'une maladie. Ce risque est d'autant plus important que l'activité est bien développée, ce qui va souvent de pair avec un nombre plus élevé de conchyliculteurs. Par ailleurs, outre les éleveurs, de nombreux courtiers ou des "affairistes » achètent des coquillages vivants, qu'ils revendent pour immersion. Les intérêts commerciaux à court terme et apparemment contradictoires des acteurs de la filière les poussent à importer, sans toujours suivre la voie légale, ce qui crée des situations à risques, dont les répercussions vont peser sur toute la filière (comme cela a été le cas avec B. ostreae).

Enfin, les pratiques d'élevage sont susceptibles de favoriser l'implantation d'une maladie et sa propagation. Des biomasses trop élevées à l'échelle d'un bassin par rapport à la capacité biotique, engendrent des croissances lentes et favorisent l'obtention d'animaux maigres sans réserves glycogéniques. L'exemple le plus significatif, à cet égard, est celui de l'huître C. angulata décimée par une iridovirose puis remplacée par C. gigas (15). Ces populations fragilisées sont probablement plus sensibles à tous les stress qu'elles subissent lors des phases d'élevage. Les fortes densités sont également nuisibles pour l'élevage ; en revanche, elles favorisent l'expression et la propagation d'agents pathogènes opportunistes, comme les herpèsvirus (30) ou l'agent de la maladie de l'anneau brun de la palourde (23), qui peuvent engendrer des pertes de cheptel.

Un dernier risque non négligeable provient des activités extérieures; en effet, le rôle de la coque et des eaux de ballastage de bateaux dans le transport et la propagation de micro-organismes, larves, juvéniles et adultes est bien connu (19).

\section{Les risques liés à l'état des connaissances sur les maladies}

Les risques liés aux maladies sont très variables selon le type d'agent pathogène (bénin, opportuniste, infectieux, très infectieux) et sont plus ou moins difficiles à quantifier selon le degré de connaissances acquises sur les maladies.

Ainsi, il peut être admis que la présence d'une maladie à déclaration obligatoire dans un pays accroit de manière significative les risques de propagation de celle-ci en cas de transfert de l'espèce hôte.

À l'opposé, l'introduction de maladies bénignes et facilement contrôlables, comme les mytilicoloses (à Mytilicola spp.) peut s'envisager, sachant que le risque restera minime. En effet, ces agents pathogènes sont déjà présents dans beaucoup de pays et, à l'exception de conditions particulières, ils n'induisent ni de pathogénie notoire pour l'hôte, ni de mortalité durable et importante. De plus, de simples modifications de la zootechnie, comme la baisse des densités, font régresser l'impact de cet agent et permettent de maintenir un faible taux de prévalence (D. Blateau, communication personnelle).

Entre ces deux cas, relativement simples en termes de décision à prendre, il existe trois autres types de situation pour lesquelles l'évaluation du risque est plus complexe.

La première conceme l'observation d'un agent pathogène, associé à un taux de mortalité important et pour lequel très peu de données bibliographiques sont disponibles $(6,30)$ Dans ce cas, soit le risque est élevé car l'agent peut être très infectieux et virulent (par exemple B. ostreae), soit le risque est faible car l'agent incriminé ne s'est exprimé effectivement que de manière ponctuelle lors de conditions environnementales particulières, comme dans le cas de rickettsioses (5)

La deuxième se rapporte à la présence, chez un hôte, d'un agent pathogène d'un genre connu mais d'espèce incertaine, par exemple Haplosporidium sp. chez C. gigas (21) ou Marteilia sp. (11) chez Cardium edule. La présence ou l'absence de données épidémiologiques, phylogénétiques et de quantification des effets induits (répétabilité, durée, etc.) seront de nature à orienter la décision.

La troisième situation est de loin la plus délicate et la plus complexe, car elle se rapporte aux maladies naissantes, non décrites dans la littérature. Les exemples apparus ces dernières années en médecine humaine et vétérinaire ont mis en lumière les difficultés liées à ces cas, surtout lorsque des décisions relatives à la prévention doivent être prises. Le problème est identique pour les maladies des mollusques, une phase dincertitude plus ou moins longue existant entre les premières observations et celles qui permettent de mieux apprécier l'importance de la maladie.

\section{Les risques liés aux pratiques de contrôle et de recherche}

Ces demiers risques peuvent se décliner en temes d'effort et de pérennité des contrôles, d'une part, et de tecinnicité d'autre part.

A ce jour, malgré le développement de plusieurs outils (sondes ADN, anticorps monoclonaux et polyclonaux) susceptibles de déboucher sur des applications (4, 9, 10, 32, 
33), il n'existe pas de méthodes reconnues et validées au plan international qui permettent de pratiquer le diagnostic en routine pour des agents pathogènes à déclaration obligatoire ou non. Malgré leur lourdeur, les seules méthodes applicables à la grande majorité des agents pathogènes et à l'étude des cas de mortalité restent les examens cytologiques et histologiques (27), auxquels il convient d'ajouter les techniques proposées pour le diagnostic de Perkinsus marinus (3).

L'effort de contrôle s'apprécie à la durée et à la solidité des plans d'échantillonnage (nombres de points par zone, nombres d'espèces contrôlées, taille des échantillons) et à la validation des méthodes de contrôle (comparaison entre méthodes, comparaison entre lecteurs, etc.).

Pour les pays de l'Union européenne, la durée jugée acceptable pour définir le statut zoosanitaire d'une zone précise a été portée à deux ans. Le maintien du statut de cette zone, lequel peut évoluer par la suite, nécessite un suivi de base permanent et une focalisation sur les cas de mortalité.

Les pays où les données historiques n'existent pas sont potentiellement des pays à risques.

\section{Prévention}

La prévention, qui ne peut se concevoir comme une protection absolue, requiert l'adoption et la reconnaissance de quelques grands principes par tous les acteurs de la filière conchylicole.

\section{Les conchyliculteurs et courtiers}

Les professionnels qui doivent s'exprimer pour manifester leurs souhaits et leurs besoins, doivent en contrepartie reellement intégrer dans leur schéma de fonctionnement une prise de conscience des risques qu'ils encourent et quils font encourir à toute leur profession lorsqu'ils transgressent certains principes et réglementations. Les principes les plus importants se rapportent, évidemment, à toutes les précautions à prendre pour effectuer des transferts, entre zones ou pays différents. Ils portent également sur le respect des mesures daménagement prises pour chaque zone d'élevage et sur le bon emploi des techniques consellées. La formation et l'éducation quasi permanentes sont réellement nécessaires pour une bonne évolution des comportements.

\section{Les réglementations}

Les réglementations élaborées avec la participation des professionnels devraient prendre en compte les spécificités des mollusques bivalves (biologie, modes et techniques d'élevage). Si la mondialisation des échanges s'est accrue, ces dernières années, le principe de subsidiarité devrait pouvoir rester une composante importante susceptible de faciliter les discussions bilaterales entre les pays qui désirent échanger leurs produits. La réglementation doit cependant sappuyer sur un tronc commun fort, reconnu et accepté par la majorité des pays, comme cela devrait être le cas par exemple des propositions faites par l'OIE. Les réglementations doivent également rester réalistes et donc applicables, notamment en ce qui concerne les divers aspects du contrôle et de la délivrance de certificats. Une bonne certification ne peut être une certification individuelle pour chaque lot destiné à des transferts à l'exportation, mais une certification à durée limitée (valide pour une campagne d'échange et résiliable à tout moment en fonction des circonstances) portant sur des zones définies d'élevage et de provenance des mollusques. Ce principe plus souple ne peut s'envisager sans un engagement de la profession pour favoriser la transparence de l'origine des produits et la certification des lots.

\section{Conclusion}

Les activités conchylicoles qui se déroulent généralement en milieu ouvert, pratiquement incontrôlable, sont des activités très fragiles et exposées aux risques de maladies. L'expérience acquise ces trente demières annees pour les maladies importantes montre bien les difficultés de contrecarrer leur évolution et de les erradiquer $(18,39)$, car elles deviennent rapidement endémiques. Si le principe de se prémunir contre l'introduction d'agents pathogènes est généralement admis. les faits mettent en lumière les difficultés rencontrées pour bâtir et surtout pour faire appliquer des réglementations réalistes, suffisamment contraignantes et souples à la fois pour être efficaces. Les risques de transfert et de propagation des maladies ayant sérieusement augmenté ces vingt demières années, avec la mondialisation des échanges, il devient particulièrement important pour les pays producteurs de pouvoir être plus efficaces afin de répondre a ces nouvelles contraintes et à ces nouveaux besoins.

Plusieurs conditions essentielles permettent de limiter au maximum ces risques : la disponibilité d'un fonds historique de données spécifiques, l'identification des besoirs de la profession et enfin l'implication de cette dernière dans la conception et dans le respect de la réglementation.

Enfin, il importe d'intensifier la recherche et les contrôles dans les pays peu affectés par les maladies des mollusques, ainsi que dans les autres pays. La création de réseaux d'échanges méthodologiques, de systèmes de validation de méthodes de diagnostic et de plans expérimentaux pour répondre aux problèmes décrits précédemment serait de nature à consolider et à développer les acquis. L'identification de laboratoires de référence dynamiques échangeant leurs données et leur savoir devrait faciliter cette construction.

\section{Remerciements}

L'auteur remercie tous les membres permanents et cooptés de la Commission de l'OIE pour les maladies des poissons, avec lesquels il a eu l'occasion de travailler dans une ambiance sympathique et de manière constructive. 